Momentary Assessment (EMA) increases self-esteem and behaviour change intentions.

Conclusion An identifiable gap exists in the evidence-base for the effectiveness of psychosocial interventions to support the wellbeing of sex workers. Available studies are weak in their design and lack generalisability beyond female street-based sex workers. Future intervention and research should better represent the diversity of workers and types of work within the sex industry. Sex workers should be engaged in the design of interventions/research in a 'by and with' rather than 'to and for' approach so that findings adequately address and respond to their actual needs.

\section{Health care services}

\subsection{WHAT MAKES SENSE? STRATEGICAL USE OF EHEALTH TECHNOLOGY TO STIMULATE SELF-CARE IN PUBLIC SEXUAL HEALTH CARE}

F Zimbile*. Rivm/Aidsfonds Soa Aids Nederland, Amsterdam, The Netherlands

\subsection{6/sextrans-2021-sti.146}

Background The 24 Sexual Health Clinics (SHCs) at the municipal public health centres covering The Netherlands provide sexual health care for key-populations and provide about 150.000 consultations annually. Adolescents 12-25 years can anonymously contact the SHCs free-of-charge for personal consultations on sexual health subjects including STI-testing. However financial restrictions result in prolonged waiting times. Moreover, specific high-incidence populations (e.g. multicultural, low SES) are insufficiently reached. The SHCs and the national sexual health institutes developed and implemented eHealth interventions to increase the accessibility and efficiency of sexual health services and to stimulate self-care.

Approach A stepped care model (SCM) approach was used to reshape Dutch public sexual health care for young people (www.sense.info). Important principle is that clients do not receive more care than is necessary. Not all clients need the same type - or level - of care: some may be helped by a online self-assessment tool in combination with a home STI testing package, others need (immediate) support by a health professional.

Outcomes/Impact The SCM approach leads to a re-organization of public sexual health care in the Netherlands for young people. The steps in the cascade refer to different types of consultations: on- and off-line, including, video-consultations and referral to (commercial) self-testing. The SCM increased the accessibility of the health system by allocating the scarce capacity of medical professionals to those who really need it and stimulated eHealth support and self-care by others.

Innovation and Significance The SCM approach proves to decrease fragmentation of sexual youth services and eHealth solutions by offering one nationwide online sexual health platform. This increases the findability of the information, and greatly enhances efficiency. It promotes self-care and relieves the workload of public health care professionals. This systematic, efficient and blended approach provides a sound basis for cooperation between SHCs and national institutes and enables developing a strategical agenda for innovations and eHealth solutions.

\subsection{EXPLORING FREDDIE: LESSONS FROM A NOVEL VIRTUAL HIV PREP CARE MODEL IN CANADA}

${ }^{1}$ T Iglesias Trombetta, ${ }^{1} \mathrm{H}$ Moloo, ${ }^{1,2} \mathrm{C}$ Shukalek ${ }^{*} .{ }^{1}$ Freddie, Canada; ${ }^{2}$ University of Calgary, Calgary, Canada

10.1136/sextrans-2021-sti.147

Background/Purpose To combat the HIV Epidemic, the concept of pre-exposure prophylaxis (PrEP) has gained considerable traction since demonstrating efficacy in 2012 and approval in Canada in 2016. Unfortunately, this HIV prevention method has not been ubiquitously taken up by those most at risk for many reasons, including difficulty in accessing care. Innovation, and necessity during the COVID pandemic, has increased the use of technology with new care models providing 100\% of PrEP care virtually.

Approach This presentation will focus on the impacts and early findings of Freddie, a novel and entirely virtual PrEP care model in Canada focused on gender and sexual minority communities. This online health program connects those most at risk of HIV transmission with affirming prescribers across multiple provinces to break down physical and social barriers to PrEP initiation and ongoing use.

Outcomes/Impact Freddie has reached the benchmark of 1,000 patients in Canada, the majority of which have never been on PrEP before, representing a quick uptake in virtual PrEP services in Canada. The observations made thus far affirm theories that inclusive virtual care models can lead to increased access to STBBI prevention methods.

Innovation and Significance Presenters will discuss the innovative components of Freddie's virtual care model, explore findings as they relate to PrEP uptake and initiation by speaking to its initial successes and challenges, as well as how it addresses PrEP access barriers in Canada. This includes Freddie's focus on LGBTQ2S + Canadians who are known to be at higher risk of HIV and historically have faced extraordinary barriers accessing sexual health care in inclusive settings. The intervention explored in this presentation continues to address such barriers through an innovative and accessible care model.

\subsection{MAINTAINING SERVICES, RESPONDING TO NEED: THE KINGSTON (ONTARIO) QUICK TEST CLINIC}

${ }^{1} \mathrm{~B}$ Stoner*, ${ }^{2}$ P Prouse, ${ }^{2} \mathrm{E}$ Nolan, ${ }^{2} \mathrm{C}$ Wowk, ${ }^{2} \mathrm{H}$ Guan. 'Queen's University, Kingston, Canada; ${ }^{2}$ KFLandA Public Health, Kingston, Canada

\subsection{6/sextrans-2021-sti.148}

Background In early 2020, routine STI clinical services ground to a halt across Canada as a result of COVID-19 shutdowns, yet the need for STI screening, testing, and treatment continued unabated. We report on an innovative model for maintaining high-volume, low-barrier STI services during the pandemic.

Approach The Quick Test Clinic was established in June, 2020 by Kingston, Frontenac and Lennox \& Addington (KFL\&A) Public Health to facilitate nucleic acid amplification testing (NAAT) for gonorrhea (GC) and chlamydia (CT). Operating two half-days per week, the clinic invited clients to complete an intake form and submit a self-collected urine or swab specimen [rectal, meatal, vaginal, pharyngeal] without seeing a healthcare provider. Results were communicated by 